\title{
MOVIMIENTOS INTERIORES Y RACIONAMIENTO ALIMENTICIO EN LA POSTGUERRA ESPAÑOLA ${ }^{1}$
}

\author{
Roque Moreno Fonseret
}

\section{RESUMEN}

La clasificación de la provincia de Alicante en cuatro zonas económicas en función del racionamiento alimenticio después de la guerra civil motivó unas diferencias injustas en la dieta, con repercusiones económicas (ocultación, estraperlismo, etc.) y demográficas, fundamentalmente movimientos de población hacia los centros urbanos y sobre todo hacia la capital. Esta emigración afectó como mínimo al 1\% de la población, pero el porcentaje fue muy superior en los municipios rurales.

\section{RÉSUMÉ}

La classification de la province d'Alicante en quatre zones économiques par le rationnement alimentaire après la guerre civile motive unes différences injustes dans la diète, avec des répercussions économiques (occultation, marché noir, etc) et démographiques, fondamentalment des mouvements de population vers les centres honnêtes et surtout vers la ville capitale. Cette émigration affecte comme minime au $1 \%$ de la population, mais le pourcentage est très supérieur dans les municipes rurales.

\section{Introducción: movilidad de la población por racionamiento}

El sistema de racionamiento alimenticio que vivió España una vez finalizada la guerra civil tuvo efectos demográficos tan importantes como desconocidos. La asignación de diferentes cupos en función de un tipo de trabajo o, sobre todo, por la mayor o menor accesibilidad a los productos agrarios intervenidos, de forma que la cantidad de alimentos distribuidos en las áreas rurales era siempre menor, provocó situaciones más o menos injustas en diferentes zonas del Estado español. En el País Valenciano, por ejemplo, los

1 Comunicación presentada a las Jornadas sobre Movimientos migratorios provocados por la guerra civil española, Universidad de Salamanca, 15-17 de diciembre de 1988, organizados por el Ministerio de Cultura, Archivo Histórico Nacional, Sección «Guerra Civil». 
pueblos clasificados como rurales no reunieron las condiciones que lógicamente debieron distinguirle como tales; ni las producciones ni los productos cultivados (frutas, hortalizas) justificaban por sí solos una diferencia tan notable en el racionamiento de los alimentos básicos (cereales, leguminosas) y la distinción provino no por ser rurales en un amplio sentido, sino por no ser industriales.

Este hecho motivó un trasvase de población desde las zonas rurales a las localidades catalogadas como industriales y fundamentalmente a la capital de la provincia, cuyas raciones solían duplicar o triplicar las asignadas al resto de la provincia²:

«Si las Delegaciones clasificadas como industriales y por lo tanto de tipo de ración más elevada que las clasificadas como rurales observaran una extraordinaria afluencia de cartillas procedentes de estas localidades, se opondran a tal fenómeno... (de esta forma) se evitarán que las cartillas de una localidad se desplacen sin causa justificada para consumo.... ${ }^{3}$

Cuantitativamente, este trasvase de población es imposible de determinar con las fuentes tradicionales (Padrones o Censos de Población), puesto que hay que tener en cuenta que el alta censal en una localidad no entrañó generalmente la baja automática en el municipio de salida ${ }^{4}$. Por ello es muy común, sobre todo entre los historiadores, desdeñar este tipo de fuentes por la alta inflación que presentan ${ }^{5}$. Por el contrario, los censos de racionamiento, por sus especiales características, permiten fijar las corrientes de desplazamiento de la población racionada y establecer conexiones acerca de la posible inflación de los censos de población en los diferentes municipios. La disponibilidad de una buena parte de la documentación generada por la Delegación Provincial de Abastecimientos nos ha permitido conocer con cierto detalle estos factores en un ámbito concreto como es el de la provincia de Alicante.

2 Para conocer con detalle el sistema de racionamiento implantado en la provincia, véase MORENO FONSERET, R.: «Racionamiento alimenticio y mercado negro en la postguerra alicantina» en AA.VV., Guerra civil y franquismo en la provincia de Alicante, Instituto de Cultura Juan Gil Albert, Alicante, 1991, pp. 119-159.

3 DELEGACIÓN DE ABASTECIMIENTOS DE ALICANTE, Boletín Informativo, n. ${ }^{\circ}$ 8, agosto de 1943, p. 1.

4 La Delegación de Abastecimientos manifestó públicamente esta práctica usual en los Ayuntamientos. En estas condiciones, el trabajo de evitar las duplicidades en los censos de racionamiento resultaba mucho más difícil. Vean, por ejemplo, el siguiente texto: «Se da con frecuencia el caso de dar de alta en el censo (de población) de una localidad personas que continuan residiendo en la de origen, con objeto de obtener una mayor cuantía en el suministro». DELEGACIÓN DE ABASTECIMIENTOS, Boletín Informativo, n. ${ }^{\circ}$ 8, agosto 1943, p. 2.

5 Efectivamente, es frecuente entre los historiadores desechar el Censo de 1940 como fuente útil para el estudio de la población española durante la guerra e inmediata postguerra. Consideran que al ser fuente originaria de derechos (obtención de cartillas de racionamiento), las duplicidades fueron muchas. Además, se alude en ocasiones al intento de minimizar las pérdidas de la guerra civil por parte de las autoridades franquistas, que inflaron las cifras no dando de baja a los fallecidos, exiliados, fugitivos o desaparecidos. Por este motivo, también son tomadas con reservas las cifras registradas en las publicaciones del Movimiento Natural de la Población Española de 1936 a 1943. Véase, en este sentido, BORJA DE RIQUER: «Efectos demográficos de la Guerra Civil en Cataluña» en Perspectiva Contemporánea, Vol. I, n. ${ }^{\circ}$ 1, octubre 1988, o SOLE I SABATE: «El cost humá de la guerra civil: Metodología per a la recerca» en $L^{\prime}$ Avenç, n. $^{\circ} 80$, marzo 1985. Considero que dichas fuentes no están tan taradas como se pretende reflejar y, tomadas con la debida cautela, pueden servir para realizar una aproximación muy indicativa del comportamiento de los diferentes factores demográficos de la etapa. 


\section{La fuente: los censos de racionamiento}

Tras la guerra civil se implantó un nuevo sistema de racionamiento en España a través de la Orden del Ministerio de Industria y Comercio del 14 de mayo de $1939^{6}$ por la que se decretó la intervención de determinados artículos alimenticios y se encargó a la CGAT de su distribución. El cálculo de los cupos de alimentos racionados obligó a la confección de un censo nominal de habitantes con el que extender las cartillas familiares y colectivas de racionamiento. Éste contempló la división de la población en varios grupos: hombre adulto, mujer adulta, niños y niñas de hasta 14 años y hombres y mujeres de más de 60 años. Posteriormente y ante la necesidad de someter el racionamiento de pan a distintas cuantías se estableció una clasificación de las cartillas familiares a efectos de racionamiento de pan en tres categorías, que habrían de determinarse conjugando la capacidad económica de cada familia ${ }^{7}$. El 30 de enero de 1941 el Ministerio de Trabajo ${ }^{8}$ ordenó la organización de Economatos por distintas empresas y hubieron de formarse los censos particulares de estos obreros. El 30 de julio de ese año se concedió a los obreros de las explotaciones mineras y sus parientes un racionamiento especial «en consonancia con los trabajos que los mineros realizan y dada la importancia que los productos obtenidos tienen para la economía nacional» y fue preciso para ello llevar a cabo la formación de un censo minero en el que se recogían los elementos de diferenciación para poder distribuir las cartillas de racionamiento.

La complejidad del sistema acentuó la tendencia a la inflación característica de un censo de habitantes que, como el descrito, era fuente originaria de derechos, en este caso, la obtención de una Cartilla de Racionamiento. Evidentemente, la carencia de alimentos estimuló el ingenio de la población que utilizó toda clase de prácticas para aumentar las pobres raciones. La duplicidad en los censos, la utilización de cartillas de difuntos, etc., llevaron a la necesidad de sustituir la cartilla familiar por una individual que permitiese un control más rígido de la población. Se dispuso como trámite previo encaminado a la adopción de este sistema la formación de un Fichero Individual de Racionamiento en todo el territorio nacional, por lo que se hizo una nueva inscripción censal de la cual se obtuvieron fichas a conservar ordenadas alfabéticamente por cada una de las Delegaciones Locales. Las Delegaciones Provinciales, además del fichero local relativo al Censo de la capital, poseyeron otro de carácter provincial, en el que se reunieron las fichas de todos los inscritos en la provincia, incluida la capital' .

Como quiera que por decreto del 6 de abril de $1943^{10}$ se dispuso la implantación de la Cartilla Individual de Racionamiento, las depuraciones en el Censo que este nuevo sistema permitía se intensificaron y, al entrar en vigor la nueva cartilla, se logró una baja de un millón de racionados (de 28.045.405 personas en mayo de 1943 a 27.071.978 en el mes siguiente), aunque no se alcanzó la cifra que para estas fechas señalaba el Censo de población (26.500.000 aproximadamente $)^{11}$. Con algunas modificaciones, este sistema subsistió prácticamente a lo largo de todo el período de racionamiento, manteniéndose unas diferencias entre los censos de población y de racionamiento similares a la reseñada.

\footnotetext{
B. O. E. del 17 de mayo de 1939.

Orden de la Presidencia del Gobierno de 15 de noviembre de 1940.

B. O. E. del 31 de enero de 1941.

9 Orden del Ministerio de Industria y Comercio del 21 de abril de 1941.

10 B. O. E. del 15 de abril de 1943.

11 COMISARÍA GENERAL DE ABASTECIMIENTOS Y TRANSPORTES, Memoria 1942-1944, Vol. I,
} p. 15 . 
La primera Tarjeta Individual tuvo una validez de seis meses. Pero a partir de enero de 1945 se convirtió en un documento permanente con validez trienal. Ello motivó la realización cada tres años de nuevas inscripciones censales en toda España mediante hojas de empadronamiento facilitas por la C.G.A.T. Buena parte de la documentación derivada de la confección efectuada a fines de 1944 ha sido conservada y ello nos permite el estudio de los aspectos señalados en el primer apartado. Esta documentación hace referencia lógicamente al proceso de depuración del censo, precisamente la parte que nos puede aportar conclusiones más significativas.

\section{La inflación censal}

Las duplicidades existentes en el Censo de Racionamiento elaborado en 1944 en la provincia de Alicante fueron 3.306, de las que 1.244 (el 37,63\%) correspondieron a dobles inscripciones realizadas en el mismo municipio ${ }^{12}$. En conjunto, representaron el $0,51 \%$ en relación al censo de población racionada en la provincia a primeros de 1944 (646.807 personas). Lógicamente, no podemos caer en una explicación simplista y pensar que dicho índice se corresponde con el nivel de inflación censal, puesto que las prácticas utilizadas para conseguir cartillas extras (que implicaron el aumento artificial del censo) fueron muy variadas y además no todas las duplicidades acabaron siendo verificadas. Establecer la proporción en que la doble duplicidad repercutió en la inflación total resulta difícil. Comprobando los datos de la Memoria Anual de la Delegación de Abastecimientos de Alicante se observa como sólo el 38,69\% de las inscripciones «ilegales» investigadas en la capital (133 duplicidades en la misma capital y 442 en otros municipios y la capital) correspondieron a dobles inscripciones, mientras que el $43,61 \%$ (648) pertenecieron a cartillas de difuntos y el $17,70 \%$ (263) a soldados en servicio activo ${ }^{13}$. La duplicidad no fue pues la causa fundamental de inflación censal y representó tan sólo dos de cada cinco casos ilegales de inscripción.

Más arriesgado resulta, por otro lado, establecer una relación entre este nivel de inflación en los censos de racionamiento y un posible aumento en los censos de habitantes. Sin embargo, teniendo en cuenta que estas dobles inscripciones entrañaron normalmente el empadronamiento en ambos municipios ${ }^{14}$, es lógico pensar que repercutieron en alguna medida en un aumento artificial de las cifras censales en los municipios afectados. El cuadro I establece, por un lado, la relación entre el número de duplicidades y la población de hecho de 1940 en los municipios mayores de 3.000 habitantes de la provincia de Alicante, y por otro, el crecimiento intercensal de estos municipios entre 1940 y 1950. Con excepciones lógicas, casi todas ellas correspondientes a localidades del Medio Vinalopó (ciudades industriales en grave crisis por falta de materias primas), los más altos porcentajes de inflación se corresponden igualmente con un mayor crecimiento intercensal. Ello es particularmente visible en los municipios del Bajo Segura, catalogados como pueblos rurales y que, en consecuencia, recibieron menores cupos alimenticios, presentando la gran mayoría de ellos un porcentaje superior al 1\% de duplicidades. Destaca, por el contrario, el escaso índice alcanzado en las comarcas de La Marina, Marquesado y Alcoiá, que son

12 Las duplicidades aparecen recogidas en el Boletín Informativo de la Delegación, nํㅗ 38 a 48.

13 DELEGACIÓN DE ABASTECIMIENTOS DE ALICANTE, Memoria Anual 1944, Alicante, 1945.

14 A partir de 1945 fue frecuente encontrar en los Boletines Informativos notas parecidas a ésta: «Cuando cualquier persona se presente en una Delegación solicitando un certificado de baja con destino a esta capital debe advertirsele que no podrá causar alta en esta Delegación más que mediante la presentación de los siguientes documentos: contrato de casa a su nombre precisamente y contrato de trabajo». 
CuAdro I

DUPLICIDADES EN LOS MUNICIPIOS ALICANTINOS

\begin{tabular}{|c|c|c|c|c|c|c|}
\hline MUNICIPIOS & $\begin{array}{l}\text { Dupl. } \\
\text { mismo muni. }\end{array}$ & $\begin{array}{l}\text { Dupl. } \\
\text { otro muni. }\end{array}$ & $\begin{array}{l}\text { Total } \\
\text { dupl. }\end{array}$ & $\begin{array}{l}\text { Población } \\
1940\end{array}$ & $\begin{array}{l}\text { Dupl/ } \\
1940\end{array}$ & $\begin{array}{l}1950 / \\
1940\end{array}$ \\
\hline ALBATERA & 20 & 34 & 54 & 4.582 & 1,39 & 6,02 \\
\hline ALCOY & 85 & 162 & 247 & 45.792 & 0,54 & $-4,17$ \\
\hline ALICANTE & 436 & 908 & 1.344 & 96.729 & 1,39 & 7,74 \\
\hline ALMORADI & 13 & 105 & 118 & 10.459 & 1,13 & 4,50 \\
\hline ALTEA & 6 & 35 & 41 & 5.829 & 0,70 & $-1,56$ \\
\hline $\mathrm{ASPE}$ & 50 & 47 & 97 & 7.812 & 1,24 & 12,26 \\
\hline BAÑERES & 2 & 9 & 11 & 3.447 & 0,32 & 8,18 \\
\hline BENEJUZAR & 2 & 26 & 28 & 3.119 & 0,90 & 13,08 \\
\hline BENISA & 7 & 40 & 47 & 6.036 & 0,78 & $-5,50$ \\
\hline CALLOSA E. & 4 & 5 & 9 & 4.101 & 0,22 & 2,14 \\
\hline CALLOSA S. & 14 & 90 & 104 & 10.599 & 0,98 & 18,90 \\
\hline CAMPELLO & 2 & 52 & 54 & 3.582 & 1,51 & 4,50 \\
\hline CASTALLA & 11 & 22 & 33 & 3.972 & 0,83 & 3,27 \\
\hline CATRAL & 7 & 26 & 33 & 3.391 & 0,97 & 14,15 \\
\hline COCENTAINA & 14 & 18 & 32 & 8.108 & 0,39 & $-0,11$ \\
\hline CREVILLENTE & 38 & 72 & 110 & 11.403 & 0,96 & 10,81 \\
\hline DENIA & 11 & 68 & 79 & 12.323 & 0,64 & $-3,76$ \\
\hline DOLORES & 7 & 63 & 70 & 5.164 & 1,35 & 4,47 \\
\hline ELCHE & 69 & 250 & 319 & 46.596 & 0,68 & 19,91 \\
\hline ELDA & 42 & 187 & 229 & 20.050 & 1,14 & 3,23 \\
\hline GATA C. & 7 & 26 & 33 & 4.227 & 0,78 & 2,27 \\
\hline GUARDAMAR & 8 & 53 & 61 & 4.703 & 1,30 & 0,02 \\
\hline IBI & 9 & 20 & 29 & 3.929 & 0,74 & 3,86 \\
\hline JAVEA & 10 & 26 & 36 & 6.136 & 0,59 & $-3,17$ \\
\hline JIJONA & 9 & 44 & 53 & 6.942 & 0,76 & $-17,47$ \\
\hline MONFORTE & 2 & 15 & 17 & 3.217 & 0,53 & $-2,61$ \\
\hline MONOVAR & 21 & 78 & 99 & 9.933 & 1,00 & 0,46 \\
\hline MUCHAMIEL & 5 & 13 & 18 & 3.344 & 0,54 & 4,33 \\
\hline MURO ALCOY & 3 & 22 & 25 & 3.788 & 0,66 & 6,31 \\
\hline NOVELDA & 5 & 72 & 77 & 10.349 & 0,74 & 2,40 \\
\hline ORIHUELA & 34 & 168 & 202 & 43.619 & 0,46 & 3,11 \\
\hline PEDREGUER & 8 & 10 & 18 & 4.658 & 0,39 & $-2,34$ \\
\hline PEGO & 8 & 17 & 25 & 8.547 & 0,29 & $-0,65$ \\
\hline PETREL & 7 & 61 & 68 & 5.506 & 1,23 & 11,60 \\
\hline PINOSO & 7 & 56 & 63 & 5.114 & 1,23 & 17,62 \\
\hline REDOVAN & 5 & 25 & 30 & 3.202 & 0,94 & 12,43 \\
\hline ROJALES & 5 & 110 & 115 & 3.895 & 2,95 & 15,81 \\
\hline SAN JUAN & 4 & 24 & 28 & 3.434 & 0,81 & 13,91 \\
\hline SAN VICENTE & 8 & 23 & 31 & 6.493 & 0,48 & 8,53 \\
\hline SANTA POLA & 21 & 58 & 79 & 5.325 & 1,48 & 9,88 \\
\hline SAX & 2 & 32 & 34 & 3.795 & 0,90 & 6,85 \\
\hline TEULADA & 0 & 9 & 9 & 3.194 & 0,28 & $-2,82$ \\
\hline TORREVIEJA & 15 & 66 & 81 & 9.274 & 0,87 & $-3,65$ \\
\hline VILLAJOYOSA & 21 & 59 & 80 & 9.412 & 0,85 & $-1,03$ \\
\hline VILLENA & 46 & 66 & 112 & 19.065 & 0,59 & 4,87 \\
\hline TOTAL & 1.244 & 2.062 & 3.306 & 607.562 & 0,54 & 4,36 \\
\hline
\end{tabular}

FuENTE: Elaboración propia a partir del Boletín de la Delegación de Abastecimientos de Alicante. 
FIGURA 1. Provincia de Alicante, 1944 (excluida la capital). Número de duplicidades censales y corrientes de desplazamiento de la población por racionamiento alimenticio.

precisamente las que menor crecimiento intercensal presentan, muy por debajo de la media provincial, y sólo explicado por una emigración real de sus habitantes, por lo general fuera de la provincia y fundamentalmente a Valencia, dada la vinculación tradicional de estas áreas con la provincia vecina. 
FIgURA 2. Corrientes de desplazamiento de la población por racionamiento alimenticio establecidas entre la provincia de Alicante y su capital, 1944.

\section{Corrientes de desplazamientos de población por racionamiento}

Más interesante resulta conocer lo que denominaremos corrientes de desplazados por racionamiento. La fuente usada recoge los lugares de doble inscripción y señala las locali- 
dades con las que el racionado mantenía vinculaciones económicas. En función de las características de los municipios afectados se pueden establecer diferentes tipos de migraciones y posibles direcciones.

El número de duplicidades afectó fundamentalmente a la ciudad de Alicante con 1.344 dobles inscripciones $(40,6 \%)$. En un principio, el carácter de la migración sería pendular: se trataría, por lo general, de personas que se desplazaron a la ciudad de Alicante para retirar los alimentos de sus ultramarinos y panaderías, y para ello debieron residir algún día de la semana en la capital. Pero, tras la obligación de poseer contrato de vivienda en propiedad y/o la de ejercer alguna actividad económica, es muy posible que terminasen por fijar su residencia en la capital, y en este caso la migración sería definitiva. La procedencia de estos desplazados (Bajo Segura y Bajo Vinalopó) acentúa esta idea, toda vez que diversos estudios padronales ${ }^{15}$ fijan en estas comarcas el punto de origen de gran parte de los inmigrantes definitivos de la ciudad. Junto a los inmigrantes del sur de la provincia destacaron, aunque en menor medida, los de las localidades cercanas a Alicante (San Juan, Muchamiel, Campello, etc.), cuyos movimientos estaban más vigilados y la doble inscripción era, en consecuencia, mucho más difícil ${ }^{16}$. Este control se hizo más efectivo desde el momento en que las Delegaciones Locales pudieron ser objeto de castigo:

«Ahora bien, teniendo en cuenta que la permanencia en la capital está en cierto modo amparada en la falta de celo de su Delegación Local, que no ha comunicado a esta Provincial, según se la tiene ordenado, hará inevitable la sanción que la Superioridad crea oportuna... $\gg^{17}$

Junto a ésta gran importancia de la capital en la movilidad espacial por racionamiento, encontramos niveles de conexión intracomarcal altos en el Bajo Segura, Medio y Bajo Vinalopó y, en menor medida, Alcoy-Muro, aprovechando la proximidad y el menor rigor con que era investigada cualquier duplicidad que no afectase a la capital.

\section{Conclusión}

La clasificación desequilibrada de la provincia de Alicante en cuatro zonas económicas a efectos de racionamiento (zonas norte y sur agrícolas, centro industrial y zona marítima) provocó diferencias en la dieta alimenticia injustas. Este hecho no sólo tuvo repercusiones económicas (ocultación, estraperlismo, etc.) sino también demográficas, concretadas fundamentalmente en importantes movimientos de población momentáneos o definitivos. El volumen de personas afectadas es difícil de determinar, pero los datos obtenidos señalan una proporción de al menos un $1 \%$ del total de la población provincial, aunque presumiblemente el porcentaje real fuese mucho mayor de lo verificado.

Lo que si puede establecerse de una manera cierta son las direcciones de esos movimientos. Ellas explican, además, la causa de los desplazamientos: la escasez de alimentos disponibles legalmente. Estas corrientes no afectarían por igual a todos los grupos de población, sino sólo a aquellas con nivel adquisitivo relativamente alto. Y ello porque además de disponer de cartillas era necesario lógicamente el dinero para comprar los alimentos y las clases más desasistidas apenas podían conseguir su ración.

15 Véase en esta misma revista MORENO, R. y QUIÑONERO, F.: «Guerra civil y migraciones en una ciudad de retaguardia: Alicante 1936-1940».

16 La vigilancia fue mucho mayor en San Juan, Campello, Muchamiel, San Vicente, Agost, Monforte, Monóvar, Elche, Crevillente, Albatera, Cox y Callosa del Segura, localidades cuyos delegados eran advertidos continuamente y las visitas de los inspectores provinciales fueron asiduas.

17 DELEGACIÓN PROVINCIAL DE ABASTECIMIENTOS, Boletín Informativo, n. ${ }^{\circ}$ 23, noviembre de 1944 . 